\title{
CHARACTERIZING COMPACT CLIFFORD SEMIGROUPS THAT EMBED INTO CONVOLUTION AND FUNCTOR-SEMIGROUPS
}

\author{
TARAS BANAKH, MATIJA CENCELJ, OLENA HRYNIV, AND DUŠAN REPOVŠ
}

\begin{abstract}
We study algebraic and topological properties of the convolution semigroups of probability measures on a topological groups and show that a compact Clifford topological semigroup $S$ embeds into the convolution semigroup $P(G)$ over some topological group $G$ if and only if $S$ embeds into the semigroup $\exp (G)$ of compact subsets of $G$ if and only if $S$ is an inverse semigroup and has zero-dimensional maximal semilattice. We also show that such a Clifford semigroup $S$ embeds into the functor-semigroup $F(G)$ over a suitable compact topological group $G$ for each weakly normal monadic functor $F$ in the category of compacta such that $F(G)$ contains a $G$-invariant element (which is an analogue of the Haar measure on $G$ ).
\end{abstract}

\section{IntRoduction}

According to [7] (and [19]) each (commutative) semigroup $S$ embeds into the global semigroup $\Gamma(G)$ over a suitable (abelian) group $G$. The global semigroup $\Gamma(G)$ over $G$ is the set of all non-empty subsets of $G$ endowed with the semigroup operation $(A, B) \mapsto A B=\{a b: a \in A, b \in B\}$. If $G$ is a topological group, then the global semigroup $\Gamma(G)$ contains a subsemigroup $\exp (G)$ consisting of all non-empty compact subsets of $G$ and carrying a natural topology which makes it a topological semigroup. This is the Vietoris topology generated by the sub-base consisting of the sets

$$
U^{+}=\{K \in \exp (G): K \subset U\} \text { and } U^{-}=\{K \in \exp (G): K \cap U \neq \emptyset\}
$$

where $U$ runs over open subsets of $G$. Endowed with the Vietoris topology the semigroup $\exp (G)$ will be referred to as the hypersemigroup over $G$ (because its underlying topological space is the hyperspace $\exp (G)$ of $G$, see [17). The problem of detecting topological semigroups embeddable into the hypersemigroups over topological groups has been considered in the literature, see [7].

This problem was resolved in [6] for the class of Clifford compact topological semigroups: such a semigroup $S$ embeds into the hypersemigroup over a topological group if and only if the set $E$ of idempotents of $S$ is a zero-dimensional commutative subsemigroup of $S$. This characterization implies the result of 8 ] that the closed interval $[0,1]$ with the operation of the minimum does not embed into the hypersemigroup over a topological group.

Date: May 9, 2022.

2010 Mathematics Subject Classification. 20M18, 20M30, 22A15, 43A05, 54B20, 54B30.

Key words and phrases. Convolution semigroup, global semigroup, hypersemigroup, Clifford semigroup, regular semigroup, topological group, Radon measure, weakly normal monadic functor. 
We recall that a semigroup $S$ is Clifford if $S$ is the union of its subgroups. We say that a topological semigroup $S_{1}$ embeds into another topological semigroup $S_{2}$ if there is a semigroup homomorphism $h: S_{1} \rightarrow S_{2}$ which is a topological embedding.

In this paper we shall apply the already mentioned result of [6] and shall characterize Clifford compact semigroups embeddable into the convolution semigroups $P(G)$ over topological groups $G$. The convolution semigroup $P(G)$ consists of probability Radon measures on $G$ and carries the *-weak topology generated by the sub-base $\{\mu \in P(G): \mu(U)>a\}$ where $a \in \mathbb{R}$ and $U$ runs over open subsets of $G$. A measure $\mu$ defined on the $\sigma$-algebra of Borel subsets of $G$ is called Radon if for every $\varepsilon>0$ there is a compact subset $K \subset G$ with $\mu(K)>1-\varepsilon$. The semigroup operation on $P(G)$ is given by the convolution measures. We recall that the convolution $\mu * \nu$ of two measures $\mu, \nu$ is the measure assigning to each bounded continuous function $f: G \rightarrow \mathbb{R}$ the value of the integral $\int_{\mu * \nu} f=\int_{\eta} \int_{\mu} f(x y) d y d x$. For more detail information on the convolution semigroups, see [12, 14.

The following theorem is the principal result of this paper.

Theorem 1.1. For any Clifford compact topological semigroup $S$ the following assertions are equivalent:

(1) $S$ embeds into the hypersemigroup $\exp (G)$ over a topological group $G$;

(2) $S$ embeds into the convolution semigroup $P(G)$ over a topological group $G$;

(3) The set $E$ of idempotents of $S$ is a zero-dimensional commutative subsemigroup of $S$.

This theorem will be applied to a characterization of Clifford compact topological semigroups embeddable into the hyperpsemigroups or convolution semigroups over topological groups $G$ belonging to certain varieties of topological groups. A class $\mathcal{G}$ of topological groups is called a variety if it is closed under arbitrary Tychonov products, and taking closed subgroups, and quotient groups by closed normal subgroups.

Theorem 1.2. Let $\mathcal{G}$ be a non-trivial variety of topological groups. For a Clifford compact topological semigroup $S$ the following assertions are equivalent:

(1) $S$ embeds into the hypersemigroup $\exp (G)$ over a topological group $G \in \mathcal{G}$;

(2) $S$ embeds into the convolution semigroup $P(G)$ over a topological group $G \in \mathcal{G}$

(3) The set $E$ of idempotents is a zero-dimensional commutative subsemigroup of $S$ and all closed subgroups of $S$ belong to the class $\mathcal{G}$.

In fact, the equivalence of the first and last statements in Theorems 1.1 and 1.2 was proved in Theorems 3 and 4 of [6] so it remains to prove the equivalence of the assertions (1) and (2). This will be done in Proposition 1.3.

We recall that a semigroup $S$ is called regular if each element $x \in S$ is regular in the sense that $x y x=x$ for some $y \in S$. An element $x \in S$ is called (uniquely) invertible if there is a (unique) element $x^{-1} \in S$ (called the inverse of $x$ ) such that $x x^{-1} x=x$ and $x^{-1} x x^{-1}=x^{-1}$. A semigroup $S$ is called inverse if each element of $S$ is uniquely invertible. By [9,1.17], [15, II.1.2] a semigroup $S$ is inverse if and only if it is regular and the set $E$ of idempotents of $S$ is a commutative subsemigroup of $S$. An inverse semigroup $S$ is Clifford if and only if $x x^{-1}=x^{-1} x$ for all $x \in S$. In this case $S$ decomposes into the union $S=\bigcup_{e \in E} H_{e}$ of the maximal subgroups $H_{e}=\left\{x \in S: x x^{-1}=e=x^{-1} x\right\}$ of $S$ parametrized by idempotents $e$ of $S$. 
We recall that a topological semigroup $S$ is called a topological inverse semigroup if $S$ is an inverse semigroup and the inversion map $(\cdot)^{-1}: S \rightarrow S,(\cdot)^{-1}: x \mapsto x^{-1}$ is continuous. The set $E$ of idempotents of a topological inverse semigroup $S$ is a closed commutative subsemigroup of $S$ called the idempotent semilattice of $S$. We say that two idempotents $e, f \in E$ are incomparable if their product ef differs from $e$ and $f$. Two elements $x, y$ of an inverse semigroup $S$ are called conjugate if $x=z y z^{-1}$ and $y=z^{-1} x z$ for some element $z \in S$. For any idempotent $e \in E$ let $\uparrow e=\{f \in E: e f=e\}$ denote the principal filter of $e$. A topological space $X$ is called totally disconnected if for any distinct points $x, y \in X$ there is a closed-andopen subset $U \subset X$ containing $x$ but not $y$.

The following proposition shows that the semigroups $\exp (G)$ and $P(G)$ over a topological group $G$ have the same regular subsemigroups (which are necessarily topological inverse semigroups). Moreover, regular subsemigroups of $\exp (G)$ or $P(G)$ have many specific topological and algebraic features.

Proposition 1.3. Let $G$ be a topological group. A topological regular semigroup $S$ embeds into $P(G)$ if and only if $S$ embeds into $\exp (G)$. If the latter happens, then

(1) $S$ is a topological inverse semigroup;

(2) The idempotent semilattice $E$ of $S$ has totally disconnected principal filters $\uparrow e, e \in E$;

(3) An element $x \in S$ is an idempotent if and only if $x^{2} x^{-1}$ is an idempotent;

(4) Any distinct conjugated idempotents of $S$ are incomparable.

This proposition allows one to construct many examples of topological regular semigroups non-embeddable into the hypersemigroups or convolution semigroups over a topological groups. The first two assertions of this proposition imply the result of 8 , to the effect that non-trivial semigroups of left (or right) zeros as well as connected topological semilattices do not embed into the hypersemigroup $\exp (G)$ over a topological group $G$. The last two assertions imply that the semigroups $\exp (G)$ and $P(G)$ do not contain Brandt semigroups and bicyclic semigroups. By a Brandt semigroup we understand a semigroup of the form $B(H, I)=I \times H \times I \cup\{0\}$ where $H$ is a group, $I$ is a non-empty set, and the product $(\alpha, h, \beta) *\left(\alpha^{\prime}, h^{\prime}, \beta^{\prime}\right)$ of two non-zero elements of $B(H, I)$ is equal to $\left(\alpha, h h^{\prime}, \beta^{\prime}\right)$ if $\beta=\alpha^{\prime}$ and 0 otherwise. A bicyclic semigroup is a semigroup generated by two elements $p, q$ with the relation $q p=1$. Brandt semigroups and byciclic semigroups play an important role in the structure theory of inverse semigroups, see [15].

In fact, the semigroups $\exp (G)$ and $P(G)$ are special cases of the so-called functor-semigroups introduced by Teleiko and Zarichnyi [17. They observed that any weakly normal monadic functor $F: \mathcal{C} o m p \rightarrow \mathcal{C} o m p$ in the category of compact Hausdorff spaces lifts to the category of compact topological semigroups, which means that for any compact topological semigroup $X$ the space $F X$ possesses a natural semigroup structure. The semigroup operation $*$ on $F X$ can be defined by the following formula

$$
a * b=F p(a \otimes b) \text { for } a, b \in F X
$$

where $p: X \times X \rightarrow X$ is the semigroup operation of $X$ and $a \otimes b \in F(X \times X)$ is the tensor product of the elements $a, b \in F X$, see [17, §3.4].

Therefore we actually consider in this paper the following general problem: 
Problem 1.4. Given a weakly normal monadic functor $F: \mathcal{C o m p} \rightarrow \mathcal{C}$ omp, find a characterization of compact (regular, inverse, Clifford) topological semigroups embeddable into the semigroup $F X$ over a compact topological group $X$. Given a compact topological group $X$ describe invertible elements and idempotents of the semigroup $F X$.

Observe that for the functors exp and $P$ the answer to the first part of this problem is given in Theorem 1.1. Functor-semigroups induced by the functors $G$ of inclusion hyperspaces and $\lambda$ of superextension have been studied in [2]-[5], [11.

In fact, Theorem 1.2 also can be partly generalized to some monadic functors $F$ (including the functors exp, $P, G$ and $\lambda$ ). Given a compact topological group $G$ let us define an element $a \in F(G)$ to be $G$-invariant if $g * a=a=a * g$ for every $g \in G$. Here we identify $G$ with a subspace of $F(G)$ (which is possible because $F$, being weakly normal, preserves singletons). A $G$-invariant element in $F(G)$ exists for the functors exp, $P, \lambda$, and $G$. For the functors exp and $P$ a $G$-invariant element on $F(G)$ is unique: it is $G \in \exp (G)$ and the Haar measure on $G$, respectively.

Theorem 1.5. Let $F: \mathcal{C}$ omp $\rightarrow$ Comp be a weakly normal monadic functor such that for every compact topological group $G$ the semigroup $F(G)$ contains a $G$-invariant element. Each Clifford compact topological inverse semigroup $S$ with zero-dimensional idempotent semilattice $E$ embeds into the functor-semigroup $F(G)$ over the compact topological group $G=\prod_{e \in E} \widetilde{H}_{e}$ where each $\widetilde{H}_{e}$ is a non-trivial compact topological group containing the maximal subgroup $H_{e} \subset S$ corresponding to an idempotent $e \in E$ of $S$.

Proof. By Theorem 3 of [6] (see also [13]), each Clifford compact topological inverse semigroup $S$ with zero-dimensional idempotent semilattice $E$ embeds into the product $\prod_{e \in E} H_{e}^{0}$, where $H_{e}^{0}$ stands for the extension of the maximal subgroup $H_{e}$ by an isolated point $0 \notin H_{e}$ such that $x 0=0 x=0$ for all $x \in H_{e}$. For every idempotent $e \in E$, fix a non-trivial compact topological group $\widetilde{H}_{e}$ containing $H_{e}$. By our hypothesis, the space $F\left(\widetilde{H}_{e}\right)$ contains an $\widetilde{H}_{e}$-invariant element $z_{e} \in F\left(\widetilde{H}_{e}\right)$. Then $H_{e}^{0}$ can be identified with the closed subsemigroup $H_{e} \cup\left\{z_{e}\right\}$ of $F\left(\widetilde{H}_{e}\right)$ and the product $\prod_{e \in E} H_{e}^{0}$ can be identified with a subsemigroup of the product $\prod_{e \in E} F\left(\widetilde{H}_{e}\right)$. By [17, p.126], the latter product can be identified with a subspace (actually a subsemigroup) of $F\left(\prod_{e \in E} \widetilde{H}_{e}\right)=F(G)$, where $G=\prod_{e \in E} \widetilde{H}_{e}$. In this way, we obtain an embedding of $S$ into $F(G)$.

As we have said, the functors $\lambda$ of superextension and $G$ of inclusion hyperspaces satisfy the hypothesis of Theorem 1.5. However, Proposition 1.3 is specific for the functor $P$ and cannot be generalized to the functors $\lambda$ or $G$.

Indeed, for the 4-element cyclic group $C_{4}$ the semigroup $\lambda\left(C_{4}\right)$ is isomorphic to the commutative inverse semigroup $C_{4} \oplus C_{2}^{1}$, where $C_{2}^{1}=C_{2} \cup\{1\}$ is the result of attaching an external unit to the 2-element cyclic group $C_{2}$, (see [2]). On the other hand, the 12-element semigroup $C_{4} \oplus C_{2}^{1}$ cannot be embedded into $\exp \left(C_{4}\right)$ because the set of regular elements of $\exp \left(C_{4}\right)$ consists of 7 elements (which are shifted subgroups of $\left.C_{4}\right)$. Also the commutative inverse semigroup $\lambda\left(C_{4}\right) \cong C_{4} \oplus C_{2}^{1}$ can be embedded into $G\left(C_{4}\right)$ (because $\lambda$ is a submonad of $G$ ) but cannot embed into $\exp \left(C_{4}\right)$. 


\section{IDEMPOTENTS AND INVERTIBLE ELEMENTS OF THE CONVOLUTION SEMIGROUPS}

In this section we prove Proposition 1.3. For each topological group $G$ the semigroups $P(G)$ and $\exp (G)$ are related via the map of the support. We recall that the support of a Radon measure $\mu \in P(G)$ is the closed subset

$$
S_{\mu}=\{x \in G: \mu(O x)>0 \text { for each neighborhood } O x \text { of } x\}
$$

of $G$. Let $2^{G}$ denote the semigroup of all non-empty closed subsets of $G$ endowed with the semigroup operation $A * B=\overline{A B}$. By

$$
\text { supp : } P(G) \rightarrow 2^{G}, \text { supp }: \mu \mapsto S_{\mu}
$$

we denote the support map.

The following proposition is well-known, see (the proof of) Theorem 1.2.1 in [12].

Proposition 2.1. Let $G$ be a topological group. For any measures $\mu, \nu \in P(G)$ the following holds: $S_{\mu * \nu}=\overline{S_{\mu} \cdot S_{\nu}}$. This means that the support map supp :P(G) $\rightarrow$ $2^{G}$ is a semigroup homomorphism.

We shall show that for any regular element $\mu$ of the convolution semigroup $P(G)$ the support $S_{\mu}$ is compact and thus belongs to the subsemigroup $\exp (G)$ of $2^{G}$. First, we characterize idempotent measures on a topological group $G$.

A measure $\mu \in P(G)$ is called an idempotent measure if $\mu * \mu=\mu$. In 1954 Wendel 20. proved that each idempotent measure on a compact topological group coincides with the Haar measure of some compact subgroup. Later, Wendel's result was generalized to locally compact groups by Pym [16] and to all topological groups by Tortrat [18. By the Haar measure on a compact topological group $G$ we understand the unique $G$-invariant probability measure on $G$. It is a classical result that such a measure exists and is unique. Thus we have the following characterization of idempotent measures on topological groups:

Proposition 2.2. A probability Radon measure $\mu \in P(G)$ on a topological group $G$ is an idempotent of the semigroup $P(G)$ if and only if $\mu$ is the Haar measure of some compact subgroup of $G$.

We shall use this proposition to describe regular elements of the convolution semigroups. To this end we apply Proposition 4 of [6] that describes regular elements of the hypersemigroups over topological groups:

Proposition 2.3 (Banakh-Hryniv). For a compact subset $K \in \exp (G)$ of a topological group $G$ the following assertions are equivalent:

(1) $K$ is a regular element of the semigroup $\exp (G)$;

(2) $K$ is uniquely invertible in $\exp (G)$;

(3) $K=H x$ for some compact subgroup $H$ of $G$ and some $x \in G$.

A similar description of regular elements holds for the convolution semigroup:

Proposition 2.4. For a measure $\mu \in P(G)$ on a topological group $G$ the following assertions are equivalent:

(1) $\mu$ is a regular element of the semigroup $P(G)$;

(2) $\mu$ uniquely invertible in $P(G)$;

(3) $\mu=\lambda * x$ for some idempotent measure $\lambda \in P(G)$ and some element $x \in G$. 
Proof. Assume that $\mu$ is a regular element of $P(G)$ and $\nu \in P(G)$ is a measure such that $\mu * \nu * \mu=\mu$. The measure $\mu * \nu$, being an idempotent of $P(G)$ coincides with the Haar measure $\lambda$ on some compact subgroup $H$ of $G$. It follows that $\overline{S_{\mu} \cdot S_{\nu}}=S_{\mu * \nu}=S_{\lambda}=H$ and hence $S_{\mu}$ and $S_{\nu}$ are compact subsets of the group $G$. Since supp : $P(G) \rightarrow 2^{G}$ is a semigroup homomorphism, we get $S_{\mu} * S_{\nu} * S_{\mu}=S_{\mu}$, which means that $S_{\mu}$ is a regular element of the $\operatorname{semigroup} \exp (G)$ and hence $S_{\mu}=\tilde{H} x$ for some compact subgroup $\tilde{H}$ and some element $x \in G$ according to Proposition 2.3

We claim that $\tilde{H}=H$. Indeed, $H \tilde{H} x=S_{\lambda} S_{\mu}=S_{\mu * \nu} S_{\mu}=S_{\mu * \nu * \mu}=S_{\mu}=\tilde{H} x$ implies that $H \subset \tilde{H}$. Next, for any point $y \in S_{\nu}$ we get

$$
\tilde{H} x y \subset \tilde{H} x S_{\nu}=S_{\mu} S_{\nu}=S_{\lambda}=H \subset \tilde{H}
$$

which yields $x y \in \tilde{H}$ and finally $H=\tilde{H}$.

Next, we show that $\mu=\lambda * x$, which is equivalent to $\lambda=\mu * x^{-1}$. Observe that $S_{\mu * x^{-1}}=S_{\mu} x^{-1}=H x x^{-1}=H$. Now the equality $\mu * x^{-1}=\lambda$ will follow as soon as we check that the measure $\mu * x^{-1}$ is $H$-invariant. Take any point $y \in H$ and note that

$$
y * \mu * x^{-1}=y * \mu * \nu * \mu * x^{-1}=y * \lambda * \mu * x^{-1}=\lambda * \mu * x^{-1}=\mu * x^{-1},
$$

which means that the measure $\mu * x^{-1}$ on $H$ is left-invariant. Since $H$ possesses a unique left-invariant probability measure $\lambda$, we conclude that $\mu=\lambda * x$.

Finally, we show that $\mu$ is uniquely invertible in $P(G)$. It suffices to check that the measure $\nu$ is equal to $x^{-1} * \lambda$ provided $\nu=\nu * \mu * \nu$. For this just observe that $S_{\nu}$ being a unique inverse of $S_{\mu}$ is equal to $x^{-1} H$. Then $S_{x * \nu}=x S_{\nu}=x x^{-1} H$. Finally, noticing that for every $y \in H$ we get

$$
x * \nu * y=x * \nu * \mu * \nu * y=x * \nu * \lambda * y=x * \nu * \lambda=x * \nu,
$$

which means that $x * \nu$ is a right invariant measure on $H$. Since $\lambda$ is the unique right-invariant measure on $H$ we also get $x * \nu=\lambda$ and hence $\nu=x^{-1} * \lambda$.

Given a semigroup $S$ we denote the set of regular elements of $S$ by $\operatorname{Reg}(S)$.

Proposition 2.5. For any topological group $G$, the support map

$$
\text { supp : } \operatorname{Reg}(P(G)) \rightarrow \operatorname{Reg}(\exp (G))
$$

is a homeomorphism.

Proof. The preceding proposition implies that the map

$$
\text { supp : } \operatorname{Reg}(P(G)) \rightarrow \operatorname{Reg}(\exp (G))
$$

is bijective. In order to check the continuity of this map, we must prove that for any open set $U \subset G$ the preimages

$$
\begin{aligned}
& \operatorname{supp}^{-1}\left(U^{+}\right)=\{\mu \in \operatorname{Reg}(P(G)): \operatorname{supp}(\mu) \subset U\} \text { and } \\
& \operatorname{supp}^{-1}\left(U^{-}\right)=\{\mu \in \operatorname{Reg}(P(G)): \operatorname{supp}(\mu) \cap U \neq \emptyset\}
\end{aligned}
$$

are open in $P(G)$. The openness of $\operatorname{supp}^{-1}\left(U^{-}\right)$follows from the observation that $\operatorname{supp}(\mu) \cap U \neq \emptyset$ if and only if $\mu(U)>0$. To see that $\operatorname{supp}^{-1}\left(U^{+}\right)$is open, fix any measure $\mu \in \operatorname{Reg}(P(G))$ with $\operatorname{supp}(\mu) \subset U$. By Proposition 2.4 $\operatorname{supp}(\mu)=H x$ for some compact subgroup $H$ of $G$ and some $x \in G$. The 
compactness of $H$ allows us to find an open neighborhood $V$ of the neutral element of $G$ such that $H V^{2} H V^{-2} H V \subset U x^{-1}$. Now consider the open neighborhood $W=\left\{\nu \in \operatorname{Reg}(P(G)): \nu(H V x)>\frac{1}{2}\right\}$ of the measure $\mu$. We claim that $W \subset \operatorname{supp}^{-1}\left(U^{+}\right)$. Indeed, given any measure $\nu \in W$ we can apply Proposition 2.4 to find an idempotent measure $\lambda$ and $y \in G$ such that $\nu=\lambda * y$. Then $\frac{1}{2}<\nu(H V x)=\lambda\left(H V x y^{-1}\right)$. We claim that $S_{\lambda} \subset H V V H$. Indeed, given an arbitrary point $z \in S_{\lambda}$ use the $S_{\lambda}$-invariance of $\lambda$ to conclude that $\lambda\left(z H V x y^{-1}\right)=$ $\lambda\left(H V x y^{-1}\right)>1 / 2$, which implies that the intersection $z H V x y^{-1} \cap H V x y^{-1}$ is non-empty which yields $z \in H V x y^{-1}\left(H V x y^{-1}\right)^{-1}=H V V H$. The inequality $\lambda\left(H V x y^{-1}\right)>1 / 2$ implies that $H V x y^{-1}$ intersects $S_{\lambda}$ and hence the set $H V V H$. Then $y \in H V^{-2} H H V x$ and $S_{\nu}=S_{\lambda} * y \subset H V^{2} H H V^{-2} H V x \subset U x^{-1} x=U$, which implies that $\nu \in \operatorname{supp}^{-1}\left(U^{+}\right)$. This completes the proof of the continuity of the map supp : $\operatorname{Reg}(P(G)) \rightarrow \operatorname{Reg}(\exp (G))$.

The proof of the continuity of the inverse map

$$
\operatorname{supp}^{-1}: \operatorname{Reg}(\exp (G)) \rightarrow \operatorname{Reg}(P(G))
$$

is even more involved. Assume that $\operatorname{supp}^{-1}$ is discontinuous at some point $K_{0} \in$ $\operatorname{Reg}(\exp (G))$. By Proposition 2.3, $K_{0}$ is a coset of some compact subgroup of $G$. After a suitable shift, we can assume that $K_{0}$ is a compact subgroup of $G$ and then $\mu_{0}=\operatorname{supp}^{-1}\left(K_{0}\right)$ is the unique Haar measure on $K_{0}$.

Since supp ${ }^{-1}$ is discontinuous at $K_{0}$, there is a neighborhood $O\left(\mu_{0}\right) \subset P(G)$ of $\mu_{0}$ such that $\operatorname{supp}^{-1}\left(O\left(K_{0}\right)\right) \not \subset O\left(\mu_{0}\right)$ for any neighborhood $O\left(K_{0}\right) \subset \operatorname{Reg}(\exp (G))$ of $K_{0}$ in $\operatorname{Reg}(\exp (G))$.

It it well-known that the topology of $G$ is generated by the left uniform structure, which is generated by bounded left-invariant pseudometrics. Each bounded leftinvariant pseudometric $\rho$ on $G$ induces a pseudometric $\hat{\rho}$ on $P(G)$ defined by

$$
\hat{\rho}\left(\mu_{1}, \mu_{2}\right)=\inf \left\{\mu(\rho): \mu \in P(G \times G) P \operatorname{pr}_{1}(\mu)=\mu_{1}, P_{p_{2}}(\mu)=\mu_{2}\right\}
$$

where $P \operatorname{pr}_{i}: P(G \times G) \rightarrow P(G)$ is the map induced by the projection $\operatorname{pr}_{i}: G \times G \rightarrow$ $G$ onto the $i$ th coordinate. By $[1, \S 4]$ or $[10,3.10]$, the topology of the space $P(G)$ is generated by the pseudometrics $\hat{\rho}$ where $\rho$ runs over all bounded left-invariant continuous pseudometrics on $G$.

Consequently, we can find a left-invariant continuous pseudometric $\rho$ on $G$ such that the neighborhood $O\left(\mu_{0}\right)$ contains the $\varepsilon_{0}$-ball $B\left(\mu_{0}, \varepsilon_{0}\right)=\left\{\mu \in P(G): \hat{\rho}\left(\mu, \mu_{0}\right)<\right.$ $\left.\varepsilon_{0}\right\}$ for some $\varepsilon_{0}>0$. Replacing $\rho$ by a larger left-invariant pseudometric, we can additionally assume that for the pseudometric space $G_{\rho}=(G, \rho)$ the map $\gamma: G_{\rho} \times G_{\rho} \rightarrow G_{\rho}, \gamma:(x, y) \mapsto x y^{-1}$, is continuous at each point $(x, y) \in K_{0} \times K_{0}$ (this follows from the fact that for each continuous left-invariant pseudometric $\rho_{1}$ on $G$ we can find a continuous left-invariant pseudometric $\rho_{2}$ on $G$ such that the map $\gamma: G_{\rho_{2}} \times G_{\rho_{2}} \rightarrow G_{\rho_{1}}$ is continuous at points of the compact subset $\left.K_{0} \times K_{0}\right)$.

The continuity and the left-invariance of the pseudometric $\rho$ implies that the set $G_{0}=\{x \in G: \rho(x, 1)=0\}$ is a closed subgroup of $G$. Let $G^{\prime}=\left\{x G_{0}: x \in G\right\}$ be the left coset space of $G$ by $G_{0}$ and $q: G \rightarrow G^{\prime}, q: x \mapsto x G_{0}$, be the quotient projection. The space $G^{\prime}=G / G_{0}$ will be considered as a $G$-space endowed with the natural left action of the group $G$. The pseudometric $\rho$ induces a continuous left-invariant metric $\rho^{\prime}$ on $G^{\prime}$ such that $\rho(x, y)=\rho^{\prime}(q(x), q(y))$ for all $x, y \in G$. So, $q:(G, \rho) \rightarrow\left(G^{\prime}, \rho^{\prime}\right)$ is an isometry. The pseudometrics $\rho$ and $\rho^{\prime}$ induce the Hausdorff pseudometrics $\rho_{H}$ and $\rho_{H}^{\prime}$ on the hyperspaces $\exp (G)$ and $\exp \left(G^{\prime}\right)$ such that the map $\exp q: \exp (G) \rightarrow \exp \left(G^{\prime}\right)$ is an isometry. Also these pseudometrics 
induce the pseudometrics $\hat{\rho}$ and $\hat{\rho}^{\prime}$ on the spaces of measures $P(G), P\left(G^{\prime}\right)$ such that the map $P q:(P(G), \hat{\rho}) \rightarrow\left(P\left(G^{\prime}\right), \hat{\rho}^{\prime}\right)$ is an isometry. The continuity of the map $\gamma: G_{\rho}^{2} \rightarrow G_{\rho}$ at $K_{0}^{2}$ implies that $\left(K_{0}, \rho\right)$ is a (not necessarily separated) topological group, $K_{0} \cap G_{0}$ is a closed normal subgroup of $K_{0}$ and hence $K_{0}^{\prime}=$ $q\left(K_{0}\right)=K_{0} / K_{0} \cap G_{0}$ has the structure of topological group. Then $\mu_{0}^{\prime}=P q\left(\mu_{0}\right)$ is a Haar measure in $K_{0}^{\prime}$.

By the choice of the neighborhood $O\left(\mu_{0}\right)$, for every $n \in \mathbb{N}$ we can find a compact set $K_{n} \in \operatorname{Reg}(\exp (G))$ such that the measure $\mu_{n}=\operatorname{supp}^{-1}\left(K_{n}\right)$ does not belong to $O\left(\mu_{0}\right)$. Then $\hat{\rho}\left(\mu_{n}, \mu_{0}\right) \geq \varepsilon_{0}$ by the choice of the pseudometric $\rho$.

For every $n \in \mathbb{N}$ let $\mu_{n}^{\prime}=P q\left(\mu_{n}\right) \in P\left(G^{\prime}\right)$, and $K_{n}^{\prime}=q\left(K_{n}\right) \in \exp \left(G^{\prime}\right)$. The convergence of the sequence $\left(K_{n}\right)$ to $K_{0}$ in the pseudometric space $\left(\exp (G), \rho_{H}\right) \mathrm{im}$ plies the convergence of the sequence $\left(K_{n}^{\prime}\right)$ to $K_{0}^{\prime}$ in the metric space $\left(\exp \left(G^{\prime}\right), \rho_{H}^{\prime}\right)$, which implies that the union $K^{\prime}=\bigcup_{n \in \omega} K_{n}^{\prime}$ is compact in the metric space $\left(G^{\prime}, \rho^{\prime}\right)$. Then the subspace $P\left(K^{\prime}\right)$ is compact in the metric space $\left(P(G), \hat{\rho}^{\prime}\right)$ and hence the sequence $\left(\mu_{n}^{\prime}\right)_{n \in \mathbb{N}}$ contains a subsequence that converges to some measure $\mu^{\prime}$ in $\left(P\left(G^{\prime}\right), \hat{\rho}^{\prime}\right)$. We lose no generality assuming that whole sequence $\left(\mu_{n}^{\prime}\right)_{n \in \mathbb{N}}$ converges to $\mu^{\prime}$. Since $\varepsilon_{0} \leq \hat{\rho}\left(\mu_{n}, \mu_{0}\right)=\hat{\rho}^{\prime}\left(\mu_{n}^{\prime}, \mu_{0}^{\prime}\right)$, we conclude that $\mu^{\prime} \neq \mu_{0}^{\prime}$. We shall derive a contradiction (with the uniqueness of a left-invariant probability measure on compact groups) by showing that $\mu^{\prime}$ is a left-invariant measure on $K_{0}^{\prime}$, distinct from the Haar measure $\mu_{0}^{\prime}$.

The $\hat{\rho}^{\prime}$-convergence $\mu_{n}^{\prime} \rightarrow \mu^{\prime}$ and $\rho_{H}^{\prime}$-convergence $\operatorname{supp}\left(\mu_{n}^{\prime}\right)=K_{n}^{\prime} \rightarrow K_{0}^{\prime}$ imply that $\operatorname{supp}\left(\mu^{\prime}\right) \subset K_{0}^{\prime}$ and thus $\mu^{\prime}$ is a probability measure on the compact topological group $K_{0}^{\prime}$. It remains to check that the measure $\mu^{\prime}$ is left-invariant. Assuming the converse, we can find a point $a \in K_{0}^{\prime}$ such that $a * \mu^{\prime} \neq \mu^{\prime}$ and thus $\varepsilon=\hat{\rho}^{\prime}\left(\mu^{\prime}, a * \mu^{\prime}\right)>$ 0 . Since the map $\gamma: G_{\rho} \times G_{\rho} \rightarrow G_{\rho}$ is continuous at each point $(x, y) \in K_{0} \times K_{0}$, we can find a positive $\delta<\frac{\varepsilon}{4}$ so small that $\rho\left(x y, x^{\prime} y\right)<\frac{\varepsilon}{4}$ for any $x, y \in K_{0}$ and $x^{\prime} \in G$ with $\rho\left(x^{\prime}, x\right)<\delta$. Since $\rho_{H}\left(K_{n}, K_{0}\right) \rightarrow 0$ and $\hat{\rho}^{\prime}\left(\mu_{n}^{\prime}, \mu^{\prime}\right) \rightarrow 0$, there is a number $n \in \mathbb{N}$ and a point $a_{n} \in K_{n}$ such that $\rho\left(a, a_{n}\right)<\delta$ and $\hat{\rho}^{\prime}\left(\mu_{n}^{\prime}, \mu^{\prime}\right) \leq \varepsilon / 4$. Consider two left shifts $l_{a}: G \rightarrow G, l_{a}: x \mapsto a x$, and $l_{a_{n}}: G \rightarrow G$. The choice of $\delta$ guarantees that $\rho_{K_{0}}\left(l_{a}, l_{a_{n}}\right)=\sup _{x \in K_{0}} \rho\left(l_{a}(x), l_{a_{n}}(x)\right) \leq \frac{\varepsilon}{4}$. Then

$$
\hat{\rho}^{\prime}\left(a * \mu^{\prime}, a_{n} * \mu^{\prime}\right)=\hat{\rho}^{\prime}\left(P l_{a}\left(\mu^{\prime}\right), P l_{a_{n}}\left(\mu^{\prime}\right)\right) \leq \frac{\varepsilon}{4} .
$$

The left shift $l_{a_{n}}: G \rightarrow G$, being an isometry of the pseudometric space $(G, \rho)$, induces an isometry $l_{a_{n}}^{\prime}: G^{\prime} \rightarrow G^{\prime}$ of the metric space $\left(G^{\prime}, \rho^{\prime}\right)$, which induces the isometry $P l_{a_{n}}^{\prime}: P\left(G^{\prime}\right) \rightarrow P\left(G^{\prime}\right)$ of the corresponding space of measures. So, $\hat{\rho}^{\prime}\left(a_{n} * \mu^{\prime}, a_{n} * \mu_{n}^{\prime}\right)=\hat{\rho}^{\prime}\left(P l_{a_{n}}^{\prime}\left(\mu^{\prime}\right), P l_{a_{n}}^{\prime}\left(\mu_{n}^{\prime}\right)\right)=\hat{\rho}^{\prime}\left(\mu^{\prime}, \mu_{n}^{\prime}\right) \leq \frac{\varepsilon}{4}$. The compact set $K_{n}$, being a regular element of the semigroup $\exp (G)$ is equal to $H_{n} x_{n}$ for some compact subgroup $H_{n} \subset G$ and some point $x_{n} \in G$ according to Proposition 2.3. Then $\mu_{n}=\operatorname{supp}^{-1}\left(K_{n}\right)$ is equal to $\lambda_{n} * x_{n}$ where $\lambda_{n}$ is the Haar measure on the group $H_{n}$. Since $\lambda_{n}$ is left-invariant, $a_{n} * \mu_{n}=a_{n} * \lambda_{n} * x_{n}=\lambda_{n} * x_{n}=\mu_{n}$ and hence $a_{n} * \mu_{n}^{\prime}=\mu_{n}^{\prime}$.

Now we see that

$$
\begin{aligned}
\hat{\rho}^{\prime}\left(\mu^{\prime}, a * \mu^{\prime}\right) & \leq \hat{\rho}^{\prime}\left(\mu^{\prime}, \mu_{n}^{\prime}\right)+\hat{\rho}^{\prime}\left(\mu_{n}^{\prime}, a_{n} * \mu_{n}^{\prime}\right)+\hat{\rho}^{\prime}\left(a_{n} * \mu_{n}^{\prime}, a_{n} * \mu^{\prime}\right)+\hat{\rho}^{\prime}\left(a_{n} * \mu^{\prime}, a * \mu^{\prime}\right) \leq \\
& \leq \frac{\varepsilon}{4}+0+\frac{\varepsilon}{4}+\frac{\varepsilon}{4}<\varepsilon=\hat{\rho}^{\prime}\left(\mu^{\prime}, a * \mu^{\prime}\right),
\end{aligned}
$$

which is a desired contradiction. 
The following corollary establishes the first part of Proposition 1.3. The second part of that proposition follows from Theorem 2 of 6 .

Corollary 2.6. Let $G$ be a topological group. Then a topological regular semigroup $S$ can be embedded into the hypersemigroup $\exp (G)$ if and only if $S$ can be embedded into the convolution semigroup $P(G)$.

Proof. If $S \subset \exp (G)$ is a regular subsemigroup, then $S \subset \operatorname{Reg}(\exp (G))$ and $\operatorname{supp}^{-1}(S)$ is an isomorphic copy of $S$ in $P(G)$ according to Propositions 2.5 Conversely, if $S \subset P(G)$ is a regular subsemigroup, then its $\operatorname{image} \operatorname{supp}(S)$ is an isomorphic copy of $S$ in $\exp (G)$.

\section{ACKNOWLEDGEMENTS}

This research was supported by the Slovenian Research Agency grants P1-02920101-04, J1-9643-0101 and J1-2057-0101. We thank the referee for comments and suggestions.

\section{REFERENCES}

[1] T. Banakh, Topology of spaces of probability measures, II, Mat. Stud. 5 (1995), 88-106 (in Russian).

[2] T. Banakh, V. Gavrylkiv, O. Nykyforchyn, Algebra in superextensions of groups, I: zeros and commutativity, Algebra Discrete Math. no.3 (2008), 1-29.

[3] T.Banakh, V.Gavrylkiv, Algebra in superextensions of groups, II: cancelativity and centers, Algebra Discrete Math. no.4 (2008), (2008), 114.

[4] T.Banakh, V.Gavrylkiv, Algebra in the superextensions of groups: minimal left ideals, Mat. Stud. 31:2 (2009) 142148.

[5] T.Banakh, V.Gavrylkiv, Algebra in the superextensions of twinic groups, Dissert. Math. 473 (2010), 74pp.

[6] T. Banakh, O. Hryniv, Embedding topological semigroups into the hyperspaces over topological groups, Acta Univ. Carolinae, Math. et Phys. 48:2 (2007), 3-18.

[7] S.G. Bershadskii, Imbeddability of semigroups in a global supersemigroup over a group, Semigroup varieties and semigroups of endomorphisms, Leningrad. Gos. Ped. Inst., Leningrad, 1979, $47-49$.

[8] R.G. Bilyeu, A. Lau, Representations into the hyperspace of a compact group, Semigroup Forum 13 (1977), 267-270.

[9] A.H. Clifford, G.B. Preston, The Algebraic Theory of Semigroups, Vol. 1, Math. Surv., No.7, Amer. Math. Soc., Providence, R.I., 1964.

[10] V.V. Fedorchuk, Functors of probability measures in topological categories, J. Math. Sci. 91:4 (1998), 3157-3204.

[11] V. Gavrylkiv, Right-topological semigroup operations on inclusion hyperspaces, Mat. Stud. 29:1 (2008), 18-34.

[12] H. Heyer, Probability Measures on Locally Compact Groups, Ergebnisse der Mathematik und ihrer Grenzgebiete, Band 94. Springer-Verlag, Berlin-New York, 1977.

[13] O. Hryniv, Universal objects in some classes of Clifford topological inverse semigroups, Semigroup Forum 75:3 (2007), 683-689.

[14] K.R. Parthasarathy, Probability Measures on Metric Spaces, Amer. Math. Soc., Providence, R.I., 2005.

[15] M. Petrich, Introduction to Semigroups, Charles E. Merrill Publishing Co., Columbus, Ohio, 1973.

[16] J.S. Pym, Idempotent measures on semigroups, Pacific J. Math. 12 (1962), 685-698.

[17] A. Teleiko, M. Zarichnyi, Categorial Topology of Compact Hausdorff Spaces, VNTL Publ., Lviv, 1999.

[18] A. Tortrat, Lois de probabilité sur un espace topologique complètement régulier et produits infinis à termes indépendants dans un groupe topologique, Ann. Inst. H. Poincaré Sect. B 1 (1964/1965), 217-237. 
[19] V. Trnkova, On a representation of commutative semigroups, Semigroup Forum 10:3 (1975), 203-214.

[20] J.G. Wendel, Haar measure and the semigroup of measures on a compact group, Proc. Amer. Math. Soc. 5 (1954), 923-929.

Instytut Matematyki, Akademia Świętokrzyska, Kielce, Poland and Department of Mathematics, Ivan Franko National University of Lviv, Lviv, Ukraine E-mail address: tbanakh@yahoo.com

Institute of Mathematics, Physics and Mechanics, and Faculty of Education, University of Ljubluana, P.O.B. 2964, Ljubluana, 1001, Slovenia

E-mail address: matija.cencelj@guest.arnes.si

Department of Mathematics, Ivan Franko National University of Lviv, Lviv, Ukraine E-mail address: olena_hryniv@ukr.net

Faculty of Mathematics and Physics, and Faculty of Education, University of LjublJana, P.O.B. 2964, Ljubluana, 1001, Slovenia

E-mail address: dusan.repovs@guest.arnes.si 